University of South Florida

DIGITAL COMMONS

Digital Commons @ University of

@ UNIVERSITY OF SOUTH FLORIDA

South Florida

$1-1-2016$

\title{
2016 Work Plan USF Tampa
}

USF

Follow this and additional works at: https://digitalcommons.usf.edu/usf_accountability_reports

\section{Scholar Commons Citation}

USF, "2016 Work Plan USF Tampa" (2016). USF Accountability Reports. 64.

https://digitalcommons.usf.edu/usf_accountability_reports/64

This Article is brought to you for free and open access by the USF Archives at Digital Commons @ University of South Florida. It has been accepted for inclusion in USF Accountability Reports by an authorized administrator of Digital Commons @ University of South Florida. For more information, please contact digitalcommons@usf.edu. 


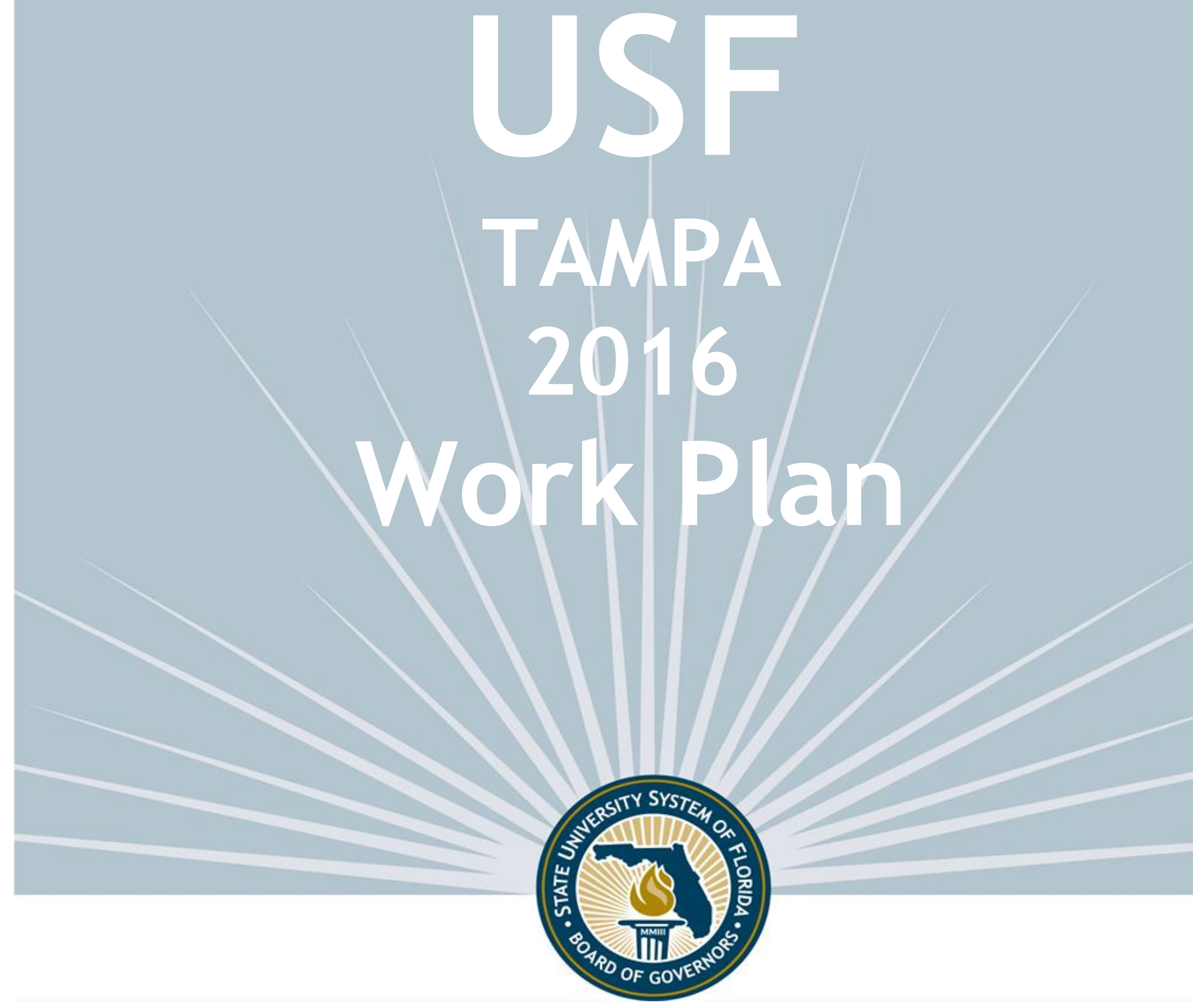

University of South Florida Tampa

University Work Plan Presentation

for Board of Governors June 2016 Meeting

STATE UNIVERSITY SYSTEM of FLORIDA $\mid$ Board of Governors 


\section{INTRODUCTION}

The State University System of Florida has developed three tools that aid in guiding the System's future.

1) The Board of Governors' 2025 System Strategic Plan is driven by goals and associated metrics that stake out where the System is headed;

2) The Board's Annual Accountability Report provides yearly tracking for how the System is progressing toward its goals;

3) Institutional Work Plans connect the two and create an opportunity for greater dialogue relative to how each institution contributes to the System's overall vision.

These three documents assist the Board with strategic planning and with setting short-, mid-and long-term goals. The Board will use these documents to help advocate for all System institutions and foster even greater coordination with the institutions and their Boards of Trustees.

Longer-term goals will inform future agendas of the Board's Strategic Planning Committee. The Board's acceptance of a work plan does not constitute approval of any particular component, nor does it supersede any necessary approval processes that may be required for each component. 


\section{TABLE OF CONTENTS}

\section{STRATEGY}
a. Mission Statement
b. Vision Statement
c. Statement of Strategy
d. Strengths and Opportunities
e. Key Initiatives \& Investments

2. PERFORMANCE BASED FUNDING METRICS

\section{PREEMINENT RESEARCH UNIVERSITY METRICS}

4. KEY PERFORMANCE INDICATORS
a. Teaching \& Learning
b. Scholarship, Research and Innovation
c. Institution Specific Goals

\section{ENROLLMENT PLANNING}

\section{ACADEMIC PROGRAM COORDINATION}

\section{STUDENT DEBT \& NET COST}

8. UNIVERSITY REVENUES

9. TUITION, FEES AND HOUSING PROJECTIONS

10. DEFINITIONS 


\section{MISSION STATEMENT (What is your purpose?)}

The University of South Florida's mission is to deliver competitive undergraduate, graduate, and professional programs, to generate knowledge, foster intellectual development, and ensure student success in a competitive global market.

\section{VISION STATEMENT (What do you aspire to?)}

The University of South Florida is a global research university dedicated to student success and positioned for membership in the Association of American Universities (AAU) and Florida preeminence.

As Florida's leading metropolitan research university, USF is dedicated to:

- Student access, learning, and success through a vibrant, interdisciplinary, and learner-centered research environment, incorporating a global curriculum.

- Research and scientific discovery that strengthens the economy, promotes civic engagement, culture, and the arts, and design, and builds sustainable communities through the generation, dissemination and translation of new knowledge across all academic and health-related disciplines.

- Partnerships that build a significant locally and globally integrated university-community collaborations through sound scholarly and artistic activities and technological innovation.

- A sustainable economic base to support USF's continued academic advancement.

\section{STATEMENT OF STRATEGY (How will you get there?)}

Given your mission, vision, strengths and available resources, provide a brief description of your market and your strategy for addressing and leading it.

USF is a Carnegie-classified Doctoral Research University, Highest Research Activity, attracting students and faculty of the highest caliber. As USF moves forward in positioning itself for AAU eligibility and preeminence, it maintains its sharply focused commitment to student success, along with embracing scholarship, research, innovation, community collaboration and economic development at all levels, including USF Health.

The foundation of USF's strategy is accountability, guided by the Board of Governors' Strategic Plan, USF's Strategic Plan and the Board of Governors' performance-funding model. By benchmarking progress to our current and aspirational peer institutions, a clear roadmap guides USF toward further excellence and, ultimately, AAU membership and preeminence.

As part of a regular review process, the USF Board of Trustees and university leadership use a detailed crosswalk to track key metrics for performance-based funding, AAU membership and preeminence. They also review strategies to most effectively expand educational access, including through distance learning. This evaluation guides decision-making for resource allocation to ensure maximum impact. 
This past year in particular, there has been a heightened focus on strategic planning for each college and administrative unit at USF - aligning with USF's Strategic Plan, which aligns with the Strategic Plan for the State University System of Florida. Guided by our Board of Trustees, this intentional thinking has revealed creative new ideas for advancing key metrics. One example can be seen in USF's efforts to reduce excess hours - which has taken on a multi-pronged approach that includes policy changes to discourage the addition of unnecessary coursework, programs or majors; hiring additional advisers; implementing personalized online tracking systems; and a comprehensive marketing campaign targeting students where they spend time the most, including web portals and social media.

This year we have also leveraged our strength in STEM education and research as part of a new USF System STEM Collaborative aimed at better connecting students in STEM with our region's workforce, which is hungry for talent. This Collaborative group, made up of representatives from USF, USF St. Petersburg, USF Sarasota-Manatee, USF Health and USF Research \& Innovation held a STEM Summit in March 2016 to hear from business leaders about their needs and perspectives on STEM education and research. This event kicked off a larger effort to better align educational offerings across the STEM fields with the workforce, staking Florida's claim as a state on STEM excellence. We hope to expand this effort to include other sister institutions in the SUS in the future.

One key partnership that exemplifies both our strength in collaboration and our leadership in research is the growing National Academy of Inventors. The NAI, founded in 2010 at USF, now includes representation from every Florida SUS institution, in addition to nearly 200 top research universities and nonprofit research institutes across the country.

Another key initiative that will build momentum in the coming months and years is the move of the Morsani College of Medicine and USF Health Heart Institute to downtown Tampa - creating unprecedented synergies and opportunities for collaboration with health professionals throughout Tampa's metropolitan core and at USF's main teaching hospital, Tampa General Hospital. 


\section{STRENGTHS AND OPPORTUNITIES (within 3 years)}

What are your core capabilities, opportunities and challenges for improvement?

One of USF's key strengths is its agility. We respond quickly to challenges, continually looking for ways to improve, and readily accept new opportunities for growth. This attitude helps us quickly direct focus to those areas that are most important to our students, the Board of Governors and other state leaders.

This is reflected in our steady gains in performance-based funding metrics, the culture of innovation that has made USF one of the most productive research universities in Florida, and our everexpanding network of business and research partners in the community. It is also evident in our recent recognition as an Emerging Preeminent institution by the Florida Legislature and Governor, pending confirmation by the Board of Governors. We are grateful for the opportunity to present our strategies to work toward the preeminence designation that reflects our research mission while working hard toward those PBF goals that strengthen our undergraduate education.

Our challenge will continue to be maintaining that momentum as we seek further budget efficiencies.

\section{KEY INITIATIVES \& INVESTMENTS (within 3 years)}

Describe your top three key initiatives for the next three years that will drive improvement in Academic Quality, Operational Efficiency, and Return on Investment.

\section{Graduate well-educated global citizens through a continued commitment to student success.}

USF's No. 1 priority remains to deliver high quality, relevant and globally informed academic programs to prepare graduates for leadership roles and workforce opportunities locally and across the world. This commitment is guided by our efforts to further improve student success through personalized advising, research opportunities at every level, and career-readiness programs. USF's commitment to this global focus through its Quality Enhancement Plan for SACS reaccreditation has gained momentum and engaged faculty, students and staff university-wide. This initiative continues to work to enhance curriculum across USF with global, cross-cultural perspectives and to ensure that every student has an opportunity for a global experience-whether through a technologyenabled global classroom, a study abroad experience, or meaningful interactions with international students and cultures.

\section{Produce high-impact research and innovation that will change lives for the better, improve health, and foster positive societal change.}

USF has escalated its efforts to recruit and retain research-productive new faculty; intensified its encouragement of undergraduate research that will contribute to an overall positive education experience and enhance student success; supported interdisciplinary initiatives that aim to solve critical problems; provided training to increase external funding; and continued to promote partnerships across the university and within the broader community. 
Last year USF was successful in its application to attain status as an Innovation and Economic Prosperity University from the Association of Public and Land-grant Universities - one of only 48 universities to receive this designation nationwide. This designation is only the beginning, however, as USF continues to engage with key community stakeholders to build relationships and improve strategies for economic development.

USF has also just graduated its inaugural class of Innovation Corps (I-Corps) startup companies after being designated as an official I-Corps site by the National Science Foundation. This public-private partnership program trains university entrepreneurs with a targeted curriculum to identify valuable product and startup company opportunities from academic research. It will continue to foster a spirit of productivity across campus as USF innovators discover new applications for their research.

\section{Create new partnerships, seek new efficiencies, and cultivate opportunities that will maintain USF's position as a highly effective economic engine for Florida.}

USF's partnerships strengthen the Tampa Bay region and Florida as part of the global landscape. USF also continually works to maximize efficiencies and use its resources in the most prudent ways possible-especially given the valued new infusion of performance-based funding investments.

USF continues to work closely with its partners in the Florida Consortium of Metropolitan Research Universities - the University of Central Florida and Florida International University. The group has enjoyed great support from national grant-makers, notably the Helios Foundation, the Helmsley Charitable Trust, and the Kresge Foundation. By sharing and developing best practices in predictive analytics, targeted support, high tech pathways, and career readiness, the Consortium will continue to add value to the student success initiative at USF. In the coming months, the Consortium plans to deepen its efforts to transform STEM education and promote internship opportunities for students in the three metropolitan regions.

Meanwhile, USF continues to build upon its own strong research enterprise, which still boasts the most patents, cumulatively over the last three years, of any state university in Florida by providing the tools and training to innovators on campus so that they are best positioned for success in the marketplace. USF continues its expansion of licensing, with 119 licenses in FY 2015 (a 31 percent increase from FY 2014), and a growing product pipeline over the last three years, with 57 USF products currently on the market. 


\section{PERFORMANCE BASED FUNDING METRICS}

\begin{tabular}{|c|c|c|c|c|c|c|}
\hline & $\begin{array}{c}2015 \\
\text { ACTUAL } \\
\end{array}$ & $\begin{array}{c}2016 \\
\text { ACTUAL } \\
\end{array}$ & $\begin{array}{c}2017 \\
\text { GOALS }\end{array}$ & $\begin{array}{c}2018 \\
\text { GOALS }\end{array}$ & $\begin{array}{c}2019 \\
\text { GOALS }\end{array}$ & $\begin{array}{c}2020 \\
\text { GOALS }\end{array}$ \\
\hline $\begin{array}{l}\text { Percent of Bachelor's Graduates } \\
\text { Enrolled or Employed }(\mathbf{\$ 2 5 , 0 0 0 + )} \\
\text { within the U.S. One Year After Graduation }\end{array}$ & $\begin{array}{l}64.9 \% \\
2012-13\end{array}$ & $\begin{array}{l}65.8 \% \\
2013-14\end{array}$ & $\begin{array}{l}66.8 \% \\
2014-15\end{array}$ & $\begin{array}{l}70.0 \% \\
2015-16\end{array}$ & $\begin{array}{l}72.0 \% \\
2016-17\end{array}$ & $\begin{array}{l}74.0 \% \\
2017-18\end{array}$ \\
\hline $\begin{array}{l}\text { Median Wages of Bachelor's } \\
\text { Graduates Employed Full-time } \\
\text { in Florida One-Year After Graduation }\end{array}$ & $\underset{2012-13}{\$ 35,300}$ & $\underset{2013-14}{\$ 36,500}$ & $\begin{array}{c}\$ 37,000 \\
2014-15\end{array}$ & $\begin{array}{c}\$ 37,500 \\
2015-16\end{array}$ & $\begin{array}{c}\$ 38,000 \\
2016-17\end{array}$ & $\underset{2017-18}{\$ 38,500}$ \\
\hline $\begin{array}{l}\text { Cost per Bachelor's Degree } \\
\text { Costs to the University }\end{array}$ & \multicolumn{4}{|c|}{ Data reported at the USF System level only. } & & \\
\hline $\begin{array}{l}\text { FTIC } 6 \text { year Graduation Rate * } \\
\text { for full- and part-time students }\end{array}$ & $\begin{array}{c}66.6 \%{ }^{1} \\
2008-14\end{array}$ & $\begin{array}{c}68.1 \%^{2} \\
2009-15\end{array}$ & $\begin{array}{l}66.3 \% \\
2010-16\end{array}$ & $\begin{array}{l}70.0 \% \\
2011-17\end{array}$ & $\begin{array}{l}72.0 \% \\
2012-18\end{array}$ & $\begin{array}{l}74.0 \% \\
2013-19\end{array}$ \\
\hline $\begin{array}{l}\text { Academic Progress Rate } * \\
\text { FTIC } 2 \text { year Retention Rate with GPA>2 }\end{array}$ & $\begin{array}{c}86.7 \%^{3} \\
2013-14\end{array}$ & $\begin{array}{c}85.6 \%{ }^{4} \\
2014-15\end{array}$ & $\begin{array}{c}86.5 \% \\
2015-16\end{array}$ & $\begin{array}{l}87.5 \% \\
2016-17\end{array}$ & $\begin{array}{c}88.5 \% \\
2017-18\end{array}$ & $\begin{array}{c}90.0 \% \\
2018-19\end{array}$ \\
\hline $\begin{array}{l}\text { Bachelor's Degrees Awarded } \\
\text { Within Programs of Strategic } \\
\text { Emphasis }\end{array}$ & $\begin{array}{l}53.5 \% \\
2013-14\end{array}$ & $\begin{array}{l}56.6 \% \\
2014-15\end{array}$ & $\begin{array}{l}57.0 \% \\
2015-16\end{array}$ & $\begin{array}{l}57.0 \% \\
2016-17\end{array}$ & $\begin{array}{l}57.0 \% \\
2017-18\end{array}$ & $\begin{array}{l}57.0 \% \\
2018-19\end{array}$ \\
\hline $\begin{array}{l}\text { University Access Rate } \\
\text { Percent of Fall Undergraduates } \\
\text { with a Pell grant }\end{array}$ & $\begin{array}{l}42.5 \% \\
\text { Fall } 2013\end{array}$ & $\begin{array}{l}41.7 \% \\
\text { Fall } 2014\end{array}$ & $\begin{array}{l}40.0 \% \\
\text { Fall } 2015\end{array}$ & $\begin{array}{l}40.0 \% \\
\text { Fall } 2016\end{array}$ & $\begin{array}{l}40.0 \% \\
\text { Fall } 2017\end{array}$ & $\begin{array}{l}40.0 \% \\
\text { Fall } 2018\end{array}$ \\
\hline $\begin{array}{l}\text { Graduate Degrees Awarded Within } \\
\text { Programs of Strategic Emphasis }\end{array}$ & $\begin{array}{l}71.5 \% \\
2013-14\end{array}$ & $\begin{array}{l}76.3 \% \\
2014-15\end{array}$ & $\begin{array}{l}76.3 \% \\
2015-16\end{array}$ & $\begin{array}{l}76.3 \% \\
2016-17\end{array}$ & $\begin{array}{l}76.3 \% \\
2017-18\end{array}$ & $\begin{array}{l}76.3 \% \\
2018-19\end{array}$ \\
\hline $\begin{array}{l}\text { BOG METRIC: } \\
\text { Percent of Bachelor's Degrees } \\
\text { Without Excess Hours }\end{array}$ & $\begin{array}{l}62.8 \% \\
2013-14\end{array}$ & $\begin{array}{l}65.1 \% \\
2014-15\end{array}$ & $\begin{array}{c}67.5 \% \\
2015-16\end{array}$ & $\begin{array}{l}70.0 \% \\
2016-17\end{array}$ & $\begin{array}{l}72.5 \% \\
2017-18\end{array}$ & $\begin{array}{c}75.0 \% \\
2018-19\end{array}$ \\
\hline $\begin{array}{l}\text { UBOT METRIC: } \\
\text { Number of Post-doctoral } \\
\text { Appointees }\end{array}$ & $\begin{array}{c}289 \\
\text { Fall } 2012\end{array}$ & $\begin{array}{l}321 \\
\text { Fall } 2013\end{array}$ & $\begin{array}{c}298 \\
\text { Fall } 2014\end{array}$ & $\begin{array}{c}277 \\
\text { Fall } 2015\end{array}$ & $\begin{array}{c}285 \\
\text { Fall } 2016\end{array}$ & $\begin{array}{l}290 \\
\text { Fall } 2017\end{array}$ \\
\hline $\begin{array}{l}\text { Note: Metrics are defined in appendix. For more inf } \\
\text { *FTIC retention/academic progress rate and } 6 \text {-Yea } \\
{ }^{1} 67.6 \% \text { of USF (Tampa) students of the } 2008 \text { coho } \\
269.1 \% \text { of USF (Tampa) students of the } 2009 \text { coho } \\
386.9 \% \text { of USF (Tampa) students of the } 2013 \text { coho } \\
{ }^{4} 85.9 \% \text { of USF (Tampa) students of the } 2014 \text { coho }\end{array}$ & $\begin{array}{l}\text { nation about th } \\
\text { aduation rate } \\
\text { graduated with } \\
\text { graduated with } \\
\text { were retained } \\
\text { were retained }\end{array}$ & $\begin{array}{l}\text { e PBF model vis } \\
\text { ercentages rep } \\
\text { in the USF Syst } \\
\text { in the USF Syst } \\
\text { vithin the USF S } \\
\text { vithin the USF S }\end{array}$ & $\begin{array}{l}\text { sit: http://www. } \\
\text { resent student } \\
\text { em } \\
\text { em } \\
\text { System }\end{array}$ & $\begin{array}{l}\text { g.edu/about/b } \\
\text { arting and end }\end{array}$ & Idget/performe & $\begin{array}{l}\text { e_funding.php } \\
\text { a). }\end{array}$ \\
\hline
\end{tabular}




\section{PREEMINENT RESEARCH UNIVERSITY FUNDING METRICS}

\begin{tabular}{|c|c|c|c|c|c|c|}
\hline & $\begin{array}{l}\text { BENCH- } \\
\text { MARKS }\end{array}$ & $\begin{array}{c}2016 \\
\text { ACTUAL }\end{array}$ & $\begin{array}{c}2017 \\
\text { GOALS }\end{array}$ & $\begin{array}{c}2018 \\
\text { GOALS }\end{array}$ & $\begin{array}{c}2019 \\
\text { GOALS }\end{array}$ & $\begin{array}{c}2020 \\
\text { GOALS }\end{array}$ \\
\hline $\begin{array}{l}\text { Average GPA and SAT Score } \\
\text { for incoming freshman in Fall semester }\end{array}$ & $\begin{array}{l}4.0 \mathrm{GPA} \\
1200 \mathrm{SAT}\end{array}$ & $\begin{array}{c}4.1 \\
1223 \\
\text { Fall } 2015\end{array}$ & $\begin{array}{c}4.0 \\
1220 \\
\text { Fall } 2016\end{array}$ & $\begin{array}{l}4.05 \\
1222 \\
\text { Fall } 2017\end{array}$ & $\begin{array}{c}4.075 \\
1224 \\
\text { Fall } 2018\end{array}$ & $\begin{array}{l}4.10 \\
1226 \\
\text { Fall } 2019\end{array}$ \\
\hline $\begin{array}{l}\text { Public University National Ranking } \\
\text { in more than one national ranking }\end{array}$ & Top 50 & $\begin{array}{c}4 \\
2016\end{array}$ & $\begin{array}{c}5 \\
2017\end{array}$ & $\begin{array}{c}5 \\
2018\end{array}$ & $\begin{array}{c}5 \\
2019\end{array}$ & $\begin{array}{c}5 \\
2020\end{array}$ \\
\hline $\begin{array}{l}\text { Freshman Retention Rate } \\
\text { Full-time, FTIC }\end{array}$ & $90 \%$ & $\begin{array}{l}88 \% \\
2014-15\end{array}$ & $\begin{array}{l}90 \% \\
2015-16\end{array}$ & $\begin{array}{l}91 \% \\
2016-17\end{array}$ & $\begin{array}{l}92 \% \\
2017-18\end{array}$ & $\begin{array}{l}93 \% \\
2018-19\end{array}$ \\
\hline $\begin{array}{l}\text { 6-year Graduation Rate } \\
\text { Full-time, FTIC }\end{array}$ & $70 \%$ & $\begin{array}{l}68 \% \\
2009-15\end{array}$ & $\begin{array}{c}66.5 \% \\
2010-16\end{array}$ & $\begin{array}{c}70.0 \% \\
2011-17\end{array}$ & $\begin{array}{l}72.0 \% \\
2012-18\end{array}$ & $\begin{array}{c}74.0 \% \\
2013-19\end{array}$ \\
\hline $\begin{array}{l}\text { National Academy } \\
\text { Memberships }\end{array}$ & 6 & $\begin{array}{c}8 \\
2016\end{array}$ & $\begin{array}{c}9 \\
2017\end{array}$ & $\begin{array}{l}10 \\
2018\end{array}$ & $\begin{array}{l}10 \\
2019\end{array}$ & $\begin{array}{l}10 \\
2020\end{array}$ \\
\hline $\begin{array}{l}\text { Science \& Engineering } \\
\text { Research Expenditures (\$M) }\end{array}$ & $\$ 200 \mathrm{M}$ & $\begin{array}{l}\$ 420 \\
2014-15\end{array}$ & $\begin{array}{l}\$ 421 \\
2015-16\end{array}$ & $\begin{array}{l}\$ 427 \\
2016-17\end{array}$ & $\begin{array}{l}\$ 434 \\
2017-18\end{array}$ & $\begin{array}{l}\$ 440 \\
2018-19\end{array}$ \\
\hline $\begin{array}{l}\text { Non-Medical Science \& Engineering } \\
\text { Research Expenditures (\$M) }\end{array}$ & $\$ 150 M$ & $\begin{array}{l}\$ 229 \\
2014-15\end{array}$ & $\begin{array}{l}\$ 230 \\
2015-16\end{array}$ & $\begin{array}{l}\$ 233 \\
2016-17\end{array}$ & $\begin{array}{l}\$ 237 \\
2017-18\end{array}$ & $\begin{array}{l}\$ 241 \\
2018-19\end{array}$ \\
\hline $\begin{array}{l}\text { National Ranking in S.T.E.M. } \\
\text { Research Expenditures } \\
\text { includes public \& private institutions }\end{array}$ & $\begin{array}{l}\text { Top } 100 \\
\text { in } 5 \text { of } 8 \\
\text { disciplines }\end{array}$ & $\begin{array}{c}7 \\
2013-14\end{array}$ & $\begin{array}{c}7 \\
2014-15\end{array}$ & $\begin{array}{c}8 \\
2015-16\end{array}$ & $\begin{array}{c}8 \\
2016-17\end{array}$ & $\begin{array}{c}8 \\
2017-18\end{array}$ \\
\hline $\begin{array}{l}\text { Patents Awarded } \\
\text { over } 3 \text { year period }\end{array}$ & 100 & $\begin{array}{c}297 \\
2013-15\end{array}$ & $\begin{array}{c}291 \\
2014-16\end{array}$ & $\begin{array}{c}273 \\
2015-17\end{array}$ & $\begin{array}{c}276 \\
2016-18\end{array}$ & $\begin{array}{c}279 \\
2017-19\end{array}$ \\
\hline $\begin{array}{l}\text { Doctoral Degrees } \\
\text { Awarded Annually }\end{array}$ & 400 & $\begin{array}{c}601 \\
2014-15\end{array}$ & $\begin{array}{c}645 \\
2015-16\end{array}$ & $\begin{array}{c}650 \\
2016-17\end{array}$ & $\begin{array}{c}655 \\
2017-18\end{array}$ & $\begin{array}{c}660 \\
2018-19\end{array}$ \\
\hline $\begin{array}{l}\text { Number of Post-Doctoral } \\
\text { Appointees }\end{array}$ & 200 & $\begin{array}{c}289 \\
\text { Fall } 2012\end{array}$ & $\begin{array}{l}321 \\
\text { Fall } 2013\end{array}$ & $\begin{array}{c}298 \\
\text { Fall } 2014\end{array}$ & $\begin{array}{l}277 \\
\text { Fall } 2015\end{array}$ & $\begin{array}{l}285 \\
\text { Fall } 2016\end{array}$ \\
\hline Endowment Size (\$M) & $\$ 500 \mathrm{M}$ & $\begin{array}{l}\$ 417 \\
2014-15\end{array}$ & $\begin{array}{l}\$ 395 \\
2015-16\end{array}$ & $\begin{array}{l}\$ 412 \\
2016-17\end{array}$ & $\begin{array}{l}\$ 432 \\
2017-18\end{array}$ & $\begin{array}{l}\$ 448 \\
2018-19\end{array}$ \\
\hline NUMBER OF METRICS ABOVE THE & CHMARK & 9 & 10 & 11 & 11 & 11 \\
\hline
\end{tabular}

Note: Metrics are defined in appendix. For more information about Preeminent state research universities, see 1001.7065 Florida Statutes. 


\section{KEY PERFORMANCE INDICATORS}

Teaching \& Learning Metrics (from 2025 System Strategic Plan that are not included in PBF or Preeminence)

\begin{tabular}{|c|c|c|c|c|c|c|}
\hline & $\begin{array}{l}2015 \\
\text { ACTUAL }\end{array}$ & $\begin{array}{l}2016 \\
\text { ACTUAL }\end{array}$ & $\begin{array}{l}2017 \\
\text { GOALS }\end{array}$ & $\begin{array}{l}2018 \\
\text { GOALS }\end{array}$ & $\begin{array}{l}2019 \\
\text { GOALS }\end{array}$ & $\begin{array}{l}2020 \\
\text { GOALS }\end{array}$ \\
\hline $\begin{array}{l}\text { 2. Freshmen in Top } 10 \% \\
\text { of Graduating High School Class }\end{array}$ & $\begin{array}{l}30.0 \% \\
\text { Fall } 2014\end{array}$ & $\begin{array}{l}34.0 \% \\
\text { Fall } 2015\end{array}$ & $\begin{array}{l}35.0 \% \\
\text { Fall } 2016\end{array}$ & $\begin{array}{l}35.0 \% \\
\text { Fall } 2017\end{array}$ & $\begin{array}{c}36.0 \% \\
\text { Fall } 2018\end{array}$ & $\begin{array}{l}36.0 \% \\
\text { Fall } 2019\end{array}$ \\
\hline $\begin{array}{l}\text { 3. Professional Licensure \& } \\
\text { Certification Exam Pass Rates } \\
\text { Above Benchmarks }\end{array}$ & $\begin{array}{l}3 \text { of } 5 \\
2013-14\end{array}$ & $\begin{array}{l}5 \text { of } 5 \\
2014-15\end{array}$ & $\begin{array}{l}6 \text { of } 6 \\
2015-16\end{array}$ & $\begin{array}{l}6 \text { of } 6 \\
2016-17\end{array}$ & $\begin{array}{l}6 \text { of } 6 \\
2017-18\end{array}$ & $\begin{array}{l}6 \text { of } 6 \\
2018-19\end{array}$ \\
\hline $\begin{array}{l}\text { 4. Time to Degree } \\
\text { Mean Years for FTICS } \\
\text { in } 120 \mathrm{hr} \text { programs }\end{array}$ & $\begin{array}{c}4.9 \\
2013-14\end{array}$ & $\begin{array}{c}4.8 \\
2014-15\end{array}$ & $\begin{array}{c}4.7 \\
2015-16\end{array}$ & $\begin{array}{c}4.5 \\
2016-17\end{array}$ & $\begin{array}{c}4.3 \\
2017-18\end{array}$ & $\begin{array}{c}4.1 \\
2018-19\end{array}$ \\
\hline $\begin{array}{l}\text { 5. Four-Year FTIC } \\
\text { Graduation Rates* } \\
\text { full- and part-time students }\end{array}$ & $\begin{array}{c}44.0 \%{ }^{1} \\
2010-14\end{array}$ & $\underset{2011-15}{51.0 \%{ }^{2}}$ & $\begin{array}{l}54.0 \% \\
2012-16\end{array}$ & $\underset{2013-17}{56.0 \%}$ & $\begin{array}{l}58.0 \% \\
2014-18\end{array}$ & $\begin{array}{l}60.0 \% \\
2015-19\end{array}$ \\
\hline $\begin{array}{l}\text { 8. Bachelor's Degrees Awarded } \\
\text { First Majors Only }\end{array}$ & $\begin{array}{c}8,079 \\
2013-14\end{array}$ & $\begin{array}{l}7,991 \\
2014-15\end{array}$ & $\begin{array}{c}7,700 \\
2015-16\end{array}$ & $\begin{array}{l}7,800 \\
2016-17\end{array}$ & $\begin{array}{c}7,900 \\
2017-18\end{array}$ & $\begin{array}{l}8,000 \\
2018-19\end{array}$ \\
\hline $\begin{array}{l}\text { 9. Graduate Degrees Awarded } \\
\text { First Majors Only }\end{array}$ & $\begin{array}{c}3,179 \\
2013-14\end{array}$ & $\begin{array}{l}3,501 \\
2014-15\end{array}$ & $\begin{array}{c}3,600 \\
2015-16\end{array}$ & $\begin{array}{l}3,675 \\
2016-17\end{array}$ & $\begin{array}{c}3,750 \\
2017-18\end{array}$ & $\begin{array}{c}3,800 \\
2018-19\end{array}$ \\
\hline $\begin{array}{l}\text { 10. Bachelor's Degrees Awarded to } \\
\text { African-American \& Hispanic } \\
\text { Students }\end{array}$ & $\begin{array}{l}29.0 \% \\
2013-14\end{array}$ & $\underset{2014-15}{31.0 \%}$ & $\begin{array}{l}31.0 \% \\
2015-16\end{array}$ & $\begin{array}{l}31.0 \% \\
2016-17\end{array}$ & $\begin{array}{l}31.0 \% \\
2017-18\end{array}$ & $\begin{array}{l}32.0 \% \\
2018-19\end{array}$ \\
\hline $\begin{array}{l}\text { 11. Adult (Aged 25+) } \\
\text { Undergraduates Enrolled }\end{array}$ & $\begin{array}{l}21.0 \% \\
\text { Fall } 2013\end{array}$ & $\begin{array}{l}21.0 \% \\
\text { Fall } 2014\end{array}$ & $\begin{array}{c}20.0 \% \\
2015-16\end{array}$ & $\begin{array}{l}20.0 \% \\
2016-17\end{array}$ & $\begin{array}{l}19.0 \% \\
2017-18\end{array}$ & $\begin{array}{c}19.0 \% \\
2018-19\end{array}$ \\
\hline $\begin{array}{l}\text { 12. Percent of Undergraduate } \\
\text { FTE in Online Courses }\end{array}$ & $\begin{array}{l}21.0 \% \\
2013-14\end{array}$ & $\begin{array}{c}24.0 \% \\
2014-15\end{array}$ & $\begin{array}{c}25.5 \% \\
2015-16\end{array}$ & $\underset{2016-17}{26.2 \%}$ & $27.0 \%$ & $\begin{array}{l}27.7 \% \\
2018-19\end{array}$ \\
\hline $\begin{array}{l}\text { 16. Percent of Bachelor's Degrees in } \\
\text { STEM \& Health }\end{array}$ & $\begin{array}{l}38.0 \% \\
2013-14\end{array}$ & $\begin{array}{c}43.0 \% \\
2014-15\end{array}$ & $\begin{array}{c}44.3 \% \\
2015-16\end{array}$ & $\begin{array}{l}45.0 \% \\
2016-17\end{array}$ & $\begin{array}{c}46.0 \% \\
2017-18\end{array}$ & $\begin{array}{c}47.0 \% \\
2018-19\end{array}$ \\
\hline $\begin{array}{l}\text { 18. Percent of Graduate Degrees in } \\
\text { STEM \& Health }\end{array}$ & $\begin{array}{l}56.0 \% \\
2013-14\end{array}$ & $\underset{2014-15}{61.0 \%}$ & $\begin{array}{l}64.0 \% \\
2015-16\end{array}$ & $\begin{array}{l}65.0 \% \\
2016-17\end{array}$ & $\begin{array}{l}66.0 \% \\
2017-18\end{array}$ & $\begin{array}{l}67.0 \% \\
2018-19\end{array}$ \\
\hline IMPROVING METRICS & & $\begin{array}{c}9 \\
\text { of } 11\end{array}$ & $\begin{array}{c}\mathbf{9} \\
\text { of } 11\end{array}$ & $\begin{array}{c}7 \\
\text { of } 11\end{array}$ & $\begin{array}{c}\mathbf{9} \\
\text { of } 11\end{array}$ & $\begin{array}{c}8 \\
\text { of } 11\end{array}$ \\
\hline
\end{tabular}




\section{KEY PERFORMANCE INDICATORS (continued)}

Scholarship, Research and Innovation Metrics (from the 2025 System Strategic Plan)

\begin{tabular}{lcc|cccc} 
& $\mathbf{2 0 1 5}$ & $\mathbf{2 0 1 6}$ & $\mathbf{2 0 1 7}$ & $\mathbf{2 0 1 8}$ & $\mathbf{2 0 1 9}$ & $\mathbf{2 0 2 0}$ \\
& ACTUAL & ACTUAL & GOALS & GOALS & GOALS & GOALS \\
\hline 20. Faculty Awards & 7 & 8 & 7 & 8 & 9 & 10 \\
& 2012 & 2013 & 2014 & 2015 & 2016 & 2017 \\
\hline 22. Total Research & $\$ 489$ & $\$ 485$ & $\$ 486$ & $\$ 501$ & $\$ 516$ & $\$ 531$ \\
Expenditures (\$M) & $2013-14$ & $2014-15$ & $2015-16$ & $2016-17$ & $2017-18$ & $2018-19$ \\
\hline 23. Research Expenditures & $60 \%$ & $55 \%$ & $56 \%$ & $57 \%$ & $58 \%$ & $59 \%$ \\
Funded from External Sources & $2013-14$ & $2014-15$ & $2015-16$ & $2016-17$ & $2017-18$ & $2018-19$ \\
\hline 25. Licenses/Options & 75 & 91 & 119 & 120 & 121 & 122 \\
Executed & $2012-13$ & $2013-14$ & $2014-15$ & $2015-16$ & $2016-17$ & $2017-18$ \\
\hline 26. Number of Start-up & 11 & 11 & 8 & 9 & 10 & 11 \\
Companies Created & $2013-14$ & $2014-15$ & $2015-16$ & $2016-17$ & $2017-18$ & $2018-19$ \\
\hline \hline & & $\mathbf{2}$ & $\mathbf{3}$ & $\mathbf{5}$ & $\mathbf{5}$ & $\mathbf{5}$ \\
\multicolumn{1}{c}{ IMPROVING METRICS } & & of 5 & of 5 & of 5 & of 5 & of 5 \\
\hline
\end{tabular}

Institution Specific Goals (optional)

To further distinguish the university's distinctive mission, the university may choose to provide additional narrative and metric goals that are based on the university's own strategic plan.

\section{Narrative Goals.}

USF's first strategic priority is student at both the undergraduate and graduate levels with Metric 1 selected and supported through the new USF System STEM collaborative initiative. As a part of our commitment to student success we continue to increase the percentage of talented, diverse students within our new FTIC cohorts (Metric 2). Our student success strategic priority also is reflected through our focus on increasing the percent of course section offered via distance and blended learning (Metric 3). As a Carnegie R1 university with a strategic goal of engaging in high impact research, USF will continue to build a sustainable research infrastructure included total research expenditures (Metric 4) and federal research expenditures (Metric 5) as defined by the National Science Foundation.

\begin{tabular}{|c|c|c|c|c|c|c|}
\hline & $\begin{array}{r}2015 \\
\text { ACTUAL }\end{array}$ & $\begin{array}{c}2016 \\
\text { ACTUAL }\end{array}$ & $\begin{array}{l}2017 \\
\text { GOALS }\end{array}$ & $\begin{array}{c}2018 \\
\text { GOALS }\end{array}$ & $\begin{array}{c}2019 \\
\text { GOALS }\end{array}$ & $\begin{array}{c}2020 \\
\text { GOALS }\end{array}$ \\
\hline $\begin{array}{l}\text { Metric \#1 } \\
\text { Graduate Degrees in Areas of Strategic } \\
\text { Emphasis }\end{array}$ & $\begin{array}{c}2,274 \\
2013-14\end{array}$ & $\begin{array}{c}2,670 \\
2014-15\end{array}$ & $\begin{array}{c}2,791 \\
2015-16\end{array}$ & $\begin{array}{c}2,850 \\
2016-17\end{array}$ & $\begin{array}{c}2,905 \\
2017-18\end{array}$ & $\begin{array}{c}2,945 \\
2018-19\end{array}$ \\
\hline $\begin{array}{l}\text { Metric \#2 } \\
\text { Freshman in Top } 10 \% \text { of Graduating High } \\
\text { School Class }\end{array}$ & $\begin{array}{l}30.0 \% \\
\text { Fall } 2014\end{array}$ & $\begin{array}{l}34.0 \% \\
\text { Fall } 2015\end{array}$ & $\begin{array}{l}35.0 \% \\
\text { Fall } 2016\end{array}$ & $\begin{array}{l}35.0 \% \\
\text { Fall } 2017\end{array}$ & $\begin{array}{l}36.0 \% \\
\text { Fall } 2018\end{array}$ & $\begin{array}{c}36.0 \% \\
\text { Fall } 2019\end{array}$ \\
\hline $\begin{array}{l}\text { Metric \#3 } \\
\text { Percent of Course Sections Offered via } \\
\text { Distance and Blended Learning }\end{array}$ & $\begin{array}{l}12.0 \% \\
2013-14\end{array}$ & $\begin{array}{r}12.0 \% \\
2014-15\end{array}$ & $\begin{array}{l}12.0 \% \\
2015-16\end{array}$ & $\begin{array}{l}12.0 \% \\
2016-17\end{array}$ & $\begin{array}{l}13.0 \% \\
2017-18\end{array}$ & $\begin{array}{l}13.0 \% \\
2018-19\end{array}$ \\
\hline $\begin{array}{l}\text { Metric \#4 } \\
\text { Total Research Expenditures }\end{array}$ & $\begin{array}{l}\$ 489 M \\
2013-14\end{array}$ & $\begin{array}{l}\$ 485 \mathrm{M} \\
2014-15\end{array}$ & $\begin{array}{l}\$ 486 \mathrm{M} \\
2015-16\end{array}$ & $\begin{array}{c}\$ 501 \\
2016-17\end{array}$ & $\begin{array}{c}\$ 516 \\
2017-18\end{array}$ & $\begin{array}{c}\$ 531 \\
2018-19\end{array}$ \\
\hline $\begin{array}{l}\text { Metric \#5 } \\
\text { Federal Research Expenditures }\end{array}$ & $\begin{array}{l}\$ 223 \mathrm{M} \\
2013-14\end{array}$ & $\begin{array}{l}\$ 218 \mathrm{M} \\
2014-15\end{array}$ & $\begin{array}{l}\$ 219 M \\
2015-16\end{array}$ & $\begin{array}{l}\$ 222 \mathrm{M} \\
2016-17\end{array}$ & $\begin{array}{l}\$ 226 \mathrm{M} \\
2018-19\end{array}$ & $\begin{array}{l}\$ 229 M \\
2019-20\end{array}$ \\
\hline
\end{tabular}


FINAL - JUNE 2016

\section{ENROLLMENT PLANNING}

Planned Headcount Enrollment by Student Type (for all students at all campuses)

\begin{tabular}{|c|c|c|c|c|c|c|c|}
\hline & $\begin{array}{c}\text { FALL } 2013 \\
\text { ACTUAL }\end{array}$ & $\begin{array}{c}\text { FALL } 2014 \\
\text { ACTUAL }\end{array}$ & $\begin{array}{c}\text { FALL } 2015 \\
\text { ACTUAL }\end{array}$ & $\begin{array}{l}\text { FALL } 2016 \\
\text { PLAN }\end{array}$ & $\begin{array}{c}\text { FALL } 2017 \\
\text { PLAN }\end{array}$ & $\begin{array}{c}\text { FALL } 2018 \\
\text { PLAN }\end{array}$ & $\begin{array}{c}\text { FALL } 2019 \\
\text { PLAN }\end{array}$ \\
\hline \multicolumn{8}{|l|}{ UNDERGRADUATE } \\
\hline FTIC & 15,469 & 15,662 & 15,823 & 15,902 & 16,021 & 16,182 & 16,194 \\
\hline AA Transfers ${ }^{1}$ & 8,615 & 8,366 & 8,199 & 8,240 & 8,302 & 8,385 & 8,490 \\
\hline Other ${ }^{2}$ & 6,303 & 6,251 & 6,265 & 6,268 & 6,171 & 6,063 & 5,881 \\
\hline Subtotal & 30,387 & 30,279 & 30,287 & 30,410 & 30,494 & 30,629 & 30,564 \\
\hline \multicolumn{8}{|l|}{ GRADUATE $^{3}$} \\
\hline Master's & 6,126 & 6,300 & 6,446 & 6,510 & 6,608 & 6,707 & 6,808 \\
\hline Research Doctoral & 2,294 & 2,226 & 2,229 & 2,232 & 2,235 & 2,238 & 2,241 \\
\hline Professional Doctoral & 1,220 & 1,379 & 1,309 & 1,239 & 1,241 & 1,244 & 1,246 \\
\hline Subtotal & 9,640 & 9,905 & 9,984 & 9,981 & 10,085 & 10,189 & 10,295 \\
\hline \multicolumn{8}{|l|}{ UNCLASSIFIED } \\
\hline H.S. Dual Enrolled & 46 & 16 & 25 & 41 & 57 & 80 & 113 \\
\hline Other ${ }^{4}$ & 1,615 & 1,865 & 1,895 & 1,900 & 1,937 & 1,974 & 2,012 \\
\hline Subtotal & 1,661 & 1,881 & 1,920 & 1,941 & 1,994 & 2,054 & 2,125 \\
\hline TOTAL & 41,688 & 42,065 & 42,191 & 42,333 & 42,573 & 42,873 & 42,985 \\
\hline
\end{tabular}

Notes: This table reports the number of students enrolled at the university by student type categories. The determination for undergraduate, graduate and unclassified is based on the institutional class level values. Unclassified refers to a student who has not yet been formally admitted into a degree program but is enrolled. The student type for undergraduates is based on the Type of Student at Time of Most Recent Admission. The student type for graduates is based on the degree that is sought and the student CIP code. (1) Includes AA Transfers from the Florida College System. (2) Undergraduate - Other includes Post-Baccalaureates who are seeking a degree. (3) Includes Medical students. (4) Unclassified - Other includes Post-Baccalaureates who are not seeking a degree.

Planned FTE Enrollment by Method of Instruction (for all students at all campuses)

\begin{tabular}{|c|c|c|c|c|c|c|c|}
\hline & $\begin{array}{l}2012-13 \\
\text { ACTUAL }\end{array}$ & $\begin{array}{l}2013-14 \\
\text { ACTUAL }\end{array}$ & $\begin{array}{l}2014-15 \\
\text { ACTUAL }\end{array}$ & $\begin{array}{l}2015-16 \\
\text { PLAN }\end{array}$ & $\begin{array}{l}2016-17 \\
\text { PLAN }\end{array}$ & $\begin{array}{c}2017-18 \\
\text { PLAN }\end{array}$ & $\begin{array}{c}2018-19 \\
\text { PLAN }\end{array}$ \\
\hline \multicolumn{8}{|l|}{ UNDERGRADUATE } \\
\hline Distance (80-100\%) & 5,732 & 5,918 & 6,830 & 7,359 & 7,580 & 7,807 & 8,041 \\
\hline Hybrid (50-79\%) & 588 & 452 & 406 & 188 & 190 & 192 & 194 \\
\hline Traditional (0-50\%) & 22,410 & 22,195 & 21,285 & 21,273 & 21,108 & 20,936 & 20,758 \\
\hline $\begin{array}{r}\text { Subtotal } \\
\text { GRADUATE }\end{array}$ & 28,729 & 28,565 & 28,521 & 28,820 & 28,878 & 28,935 & 28,993 \\
\hline Distance (80-100\%) & 1,563 & 1,611 & 1,803 & 1,851 & 1,907 & 1,964 & 2,023 \\
\hline Hybrid (50-79\%) & 210 & 224 & 180 & 63 & 64 & 64 & 65 \\
\hline Traditional (0-50\%) & 5,674 & 5,884 & 6,004 & 6,159 & 6,248 & 6,338 & 6,429 \\
\hline Subtotal & 7,447 & 7,719 & 7,987 & 8,073 & 8,218 & 8,366 & 8,517 \\
\hline
\end{tabular}

Note: Full-time Equivalent (FTE) student is a measure of instructional activity that is based on the number of credit hours that students enroll. FTE is based on the standard national definition, which divides undergraduate credit hours by 30 and graduate credit hours by 24 . Distance Learning is a course in which at least 80 percent of the direct instruction of the course is delivered using some form of technology when the student and instructor are separated by time or space, or both (per $1009.24(17)$, F.S.). Hybrid is a course where $50 \%$ to $79 \%$ of the instruction is delivered using some form of technology, when the student and instructor are separated by time or space, or both (per SUDS data element 2052). Traditional refers to primarily face to face instruction utilizing some form of technology for delivery of supplemental course materials for no more than $49 \%$ of instruction (per SUDS data element 2052).

Data Provided by USF at the institution level 


\section{ENROLLMENT PLANNING (continued)}

\section{Planned FTE Enrollment Plan by Student Level}

\begin{tabular}{|c|c|c|c|c|c|c|c|c|c|}
\hline & $\begin{array}{l}2014-15 \\
\text { ACTUAL } \\
\end{array}$ & $\begin{array}{c}2015-16 \\
\text { ESTIMATE }\end{array}$ & $\begin{array}{c}2016-17 \\
\text { PLAN }\end{array}$ & $\begin{array}{c}2017-18 \\
\text { PLAN }\end{array}$ & $\begin{array}{c}2018-19 \\
\text { PLAN }\end{array}$ & $\begin{array}{c}2019-20 \\
\text { PLAN }\end{array}$ & $\begin{array}{c}2020-21 \\
\text { PLAN }\end{array}$ & $\begin{array}{c}2021-22 \\
\text { PLAN } \\
\end{array}$ & $\begin{array}{c}\text { Annual } \\
\text { Growth } \\
\text { Rate }^{*}\end{array}$ \\
\hline \multicolumn{10}{|c|}{ STATE FUNDABLE } \\
\hline \multicolumn{10}{|l|}{ RESIDENT } \\
\hline LOWER & 10,096 & 10,039 & 10,069 & 10,099 & 10,129 & 10,160 & 10,190 & 10,221 & $0.3 \%$ \\
\hline UPPER & 15,307 & 15,184 & 15,215 & 15,245 & 15,276 & 15,306 & 15,337 & 15,367 & $0.2 \%$ \\
\hline GRAD I & 3,972 & 3,815 & 3,884 & 3,954 & 4,025 & 4,098 & 4,171 & 4,246 & $1.8 \%$ \\
\hline GRAD ॥ & 1,256 & 1,153 & 1,174 & 1,195 & 1,217 & 1,239 & 1,261 & 1,284 & $1.8 \%$ \\
\hline TOTAL & 30,631 & 30,192 & 30,342 & 30,494 & 30,647 & 30,802 & 30,960 & 31,119 & $0.5 \%$ \\
\hline \multicolumn{10}{|c|}{ NON RESIDENT } \\
\hline LOWER & 1,110 & 1,331 & 1,333 & 1,336 & 1,339 & 1,341 & 1,344 & 1,347 & $0.2 \%$ \\
\hline UPPER & 1,067 & 1,326 & 1,329 & 1,331 & 1,334 & 1,337 & 1,339 & 1,342 & $0.2 \%$ \\
\hline GRAD I & 1,206 & 1,443 & 1,469 & 1,496 & 1,523 & 1,550 & 1,578 & 1,606 & $1.8 \%$ \\
\hline GRAD II & 877 & 925 & 942 & 959 & 976 & 994 & 1,011 & 1,030 & $1.8 \%$ \\
\hline TOTAL & 4,260 & 5,025 & 5,073 & 5,122 & 5,171 & 5,222 & 5,273 & 5,325 & $1.0 \%$ \\
\hline \multicolumn{10}{|l|}{ TOTAL } \\
\hline LOWER & 11,205 & 11,370 & 11,402 & 11,435 & 11,468 & 11,501 & 11,534 & 11,568 & $0.3 \%$ \\
\hline UPPER & 16,374 & 16,510 & 16,543 & 16,576 & 16,610 & 16,643 & 16,676 & 16,709 & $0.2 \%$ \\
\hline GRAD I & 5,178 & 5,259 & 5,353 & 5,450 & 5,548 & 5,648 & 5,749 & 5,853 & $1.8 \%$ \\
\hline GRAD ॥ & 2,133 & 2,079 & 2,116 & 2,154 & 2,193 & 2,232 & 2,272 & 2,313 & $1.8 \%$ \\
\hline TOTAL & 34,891 & 35,217 & 35,415 & 35,615 & 35,818 & 36,024 & 36,232 & 36,443 & $0.6 \%$ \\
\hline \multicolumn{10}{|c|}{ NOT STATE FUNDABLE } \\
\hline LOWER & 578 & 588 & 589 & 570 & 561 & 552 & 543 & 534 & $-1.9 \%$ \\
\hline UPPER & 364 & 352 & 353 & 353 & 354 & 355 & 356 & 356 & $0.2 \%$ \\
\hline GRAD I & 651 & 677 & 690 & 702 & 715 & 728 & 741 & 754 & $1.8 \%$ \\
\hline GRAD ॥ & 24 & 58 & 59 & 60 & 61 & 62 & 63 & 65 & $2.0 \%$ \\
\hline TOTAL & 1,617 & 1,675 & 1,691 & 1,685 & 1,691 & 1,697 & 1,703 & 1,709 & $0.2 \%$ \\
\hline
\end{tabular}

Note: Full-time Equivalent (FTE) student is a measure of instructional activity that is based on the number of credit hours that students enroll. FTE is based on the standard national definition, which divides undergraduate credit hours by 30 and graduate credit hours by 24 . Note*: The Planned Annual Growth Rate is a compounded rate based on the following formula: (2021-22 value divided by the 2016-17 value) to the (1/5) exponent minus one.

Medical Student Headcount Enrollments (E\&G Funded)

\begin{tabular}{lccccccccc} 
& $\begin{array}{l}2014-15 \\
\text { ACTUAL }\end{array}$ & $\begin{array}{c}2015-16 \\
\text { ESTIMATE }\end{array}$ & $\begin{array}{c}2016-17 \\
\text { PLAN }\end{array}$ & $\begin{array}{c}2017-18 \\
\text { PLAN }\end{array}$ & $\begin{array}{c}2018-19 \\
\text { PLAN }\end{array}$ & $\begin{array}{c}2019-20 \\
\text { PLAN }\end{array}$ & $\begin{array}{c}2020-21 \\
\text { PLAN }\end{array}$ & $\begin{array}{c}2021-22 \\
\text { PLAN }\end{array}$ & $\begin{array}{c}\text { Annual } \\
\text { Growth } \\
\text { Rate }\end{array}$ \\
\hline MEDICAL DOCTORATES & & & & & & & & & \\
\hline RESIDENT & 438 & 430 & 415 & 415 & 413 & 406 & 406 & 406 & $-0.4 \%$ \\
NON-RESIDENT & 53 & 74 & 74 & 74 & 74 & 74 & 74 & 74 & $0.0 \%$ \\
\hline TOTAL & 491 & 504 & 489 & 489 & 487 & 480 & 480 & 480 & $-0.4 \%$
\end{tabular}

Data Provided by USF at the institution level 


\section{ACADEMIC PROGRAM COORDINATION}

\section{New Programs For Consideration by University in AY 2016-17}

The S.U.S. Council of Academic Vice Presidents (CAVP) Academic Program Coordination Work Group will review these programs as part of their on-going coordination efforts. The programs listed below are based on the 2015 Work Plan list for programs under consideration for 2016-17.

\begin{tabular}{|c|c|c|c|c|c|c|}
\hline PROGRAM TITLES & $\begin{array}{l}\text { CIP CODE } \\
\text { 6-digit }\end{array}$ & $\begin{array}{l}\text { AREA OF } \\
\text { STRATEGIC } \\
\text { EMPHASIS }\end{array}$ & $\begin{array}{c}\text { OTHER } \\
\text { UNIVERSITIES } \\
\text { WITH SAME } \\
\text { PROGRAM }\end{array}$ & $\begin{array}{l}\text { OFFERED VIA } \\
\text { DISTANCE } \\
\text { LEARNING } \\
\text { IN SYSTEM }\end{array}$ & $\begin{array}{l}\text { PROJECTED } \\
\text { ENROLLMENT } \\
\text { in 5th year }\end{array}$ & $\begin{array}{l}\text { PROPOSED } \\
\text { DATE OF } \\
\text { SUBMISSION } \\
\text { TO UBOT }\end{array}$ \\
\hline \multicolumn{7}{|l|}{ BACHELOR'S PROGRAMS } \\
\hline $\begin{array}{l}\text { BS Public Relations, Advertising, } \\
\text { And Applied Communications }\end{array}$ & 09.0900 & GAP & FSU & $25 \%$ & 580 & Fall 2016 \\
\hline
\end{tabular}

\section{MASTER'S, SPECIALIST AND OTHER ADVANCED MASTER'S PROGRAMS}

\begin{tabular}{lllll}
\hline MS Public Relations, Advertising, 09.0900 & GAP & FSU, UNF & $0 \%$ & 68
\end{tabular}

\section{DOCTORAL PROGRAMS}

New Programs For Consideration by University in 2017-19

These programs will be used in the 2017 Work Plan list for programs under consideration for 2017-18.

\begin{tabular}{|c|c|c|c|c|c|c|}
\hline & CIP CODE & $\begin{array}{l}\text { AREA OF } \\
\text { STRATEGIC }\end{array}$ & $\begin{array}{c}\text { OTHER } \\
\text { UNIVERSITIES } \\
\text { WITH SAME }\end{array}$ & $\begin{array}{c}\text { OFFERED VIA } \\
\text { DISTANCE } \\
\text { LEARNING }\end{array}$ & $\begin{array}{c}\text { PROJECTED } \\
\text { ENROLLMENT }\end{array}$ & $\begin{array}{l}\text { PROPOSED } \\
\text { DATE OF } \\
\text { SUBMISSION }\end{array}$ \\
\hline PROGRAM TITLES & 6-digit & EMPHASIS & PROGRAM & IN SYSTEM & in 5th year & TO UBOT \\
\hline
\end{tabular}

\section{MASTER'S, SPECIALIST AND OTHER ADVANCED MASTER'S PROGRAMS}

\begin{tabular}{llccccc}
\hline DOCTORAL PROGRAMS & \multicolumn{1}{l}{} \\
\hline OTD Occupational Therapy & 51.2306 & HEALTH & - & $0 \%$ & 80 & TBD \\
\hline PhD Pharmacy & 51.2099 & STEM & FAMU, UF & $30 \%$ & 5 & TBD
\end{tabular}




\section{STUDENT DEBT \& NET COST}

\section{Student Debt Summary}

\begin{tabular}{lccccc} 
& $2010-11$ & $2011-12$ & $2012-13$ & $2013-14$ & $2014-15$ \\
\hline Percent of Bachelor's Recipients with Debt & $53 \%$ & $57 \%$ & $59 \%$ & $60 \%$ & $60 \%$ \\
\hline $\begin{array}{l}\text { Average Amount of Debt } \\
\text { for Bachelor's who have graduated with debt }\end{array}$ & $\$ 21,780$ & $\$ 22,620$ & $\$ 22,720$ & $\$ 22,720$ & $\$ 22,899$ \\
NSLDS Cohort Year & & & & & $2012-15$ \\
Student Loan Cohort Default Rate (3rd Year) & $10.1 \%$ & $9.0 \%$ & $7.5 \%$ & $5.3 \%$ & $5.2 \%$
\end{tabular}

Cost of Attendance (for Full-Time Undergraduate Florida Residents in the Fall and Spring of 2015-16)

\begin{tabular}{ccccccc} 
& $\begin{array}{c}\text { TUITION } \\
\& \text { FEES }\end{array}$ & $\begin{array}{c}\text { BOOKS } \& \\
\text { SUPPLIES }\end{array}$ & $\begin{array}{c}\text { ROOM } \\
\& \text { BOARD }\end{array}$ & TRANSPORTATION & $\begin{array}{c}\text { OTHER } \\
\text { EXPENSES }\end{array}$ & TOTAL \\
\hline ON-CAMPUS & $\$ 6,410$ & $\$ 1,200$ & $\$ 9,400$ & $\$ 1,600$ & $\$ 2,500$ & $\$ 21,110$ \\
\hline AT HOME & $\$ 6,410$ & $\$ 1,200$ & $\$ 4,700$ & $\$ 1,600$ & $\$ 2,500$ & $\$ 16,410$ \\
\hline
\end{tabular}

Estimated Net Cost by Family Income (for Full-Time Undergraduate Florida Residents in the Fall and Spring of 2015-16)

\begin{tabular}{|c|c|c|c|c|c|c|}
\hline \multirow{2}{*}{$\begin{array}{l}\text { FAMILY } \\
\text { INCOME } \\
\text { GROUPS }\end{array}$} & \multicolumn{2}{|c|}{$\begin{array}{l}\text { FULL-TIME RESIDENT } \\
\text { UNDERGRADUATES }\end{array}$} & \multirow{2}{*}{$\begin{array}{c}\text { AVG. NET } \\
\text { COST OF } \\
\text { ATTENDANCE }\end{array}$} & \multirow{2}{*}{$\begin{array}{c}\text { AVG. NET } \\
\text { TUITION } \\
\text { \& FEES }\end{array}$} & \multirow{2}{*}{$\begin{array}{c}\text { AVG. } \\
\text { GIFT AID } \\
\text { AMOUNT }\end{array}$} & \multirow{2}{*}{$\begin{array}{c}\text { AVG. } \\
\text { LOAN } \\
\text { AMOUNT }\end{array}$} \\
\hline & $\begin{array}{l}\text { UNDERG } \\
\text { HEADCOUNT }\end{array}$ & $\begin{array}{l}\text { UATES } \\
\text { PERCENT }\end{array}$ & & & & \\
\hline Below $\$ 40,000$ & 5,551 & $32 \%$ & $\$ 11,188$ & $-\$ 3,171$ & $\$ 9,059$ & $\$ 3,936$ \\
\hline$\$ 40,000-\$ 59,999$ & 2,009 & $12 \%$ & $\$ 13,290$ & $-\$ 956$ & $\$ 6,904$ & $\$ 3,802$ \\
\hline$\$ 60,000-\$ 79,999$ & 1,529 & $9 \%$ & $\$ 15,440$ & $\$ 1,382$ & $\$ 4,643$ & $\$ 4,458$ \\
\hline$\$ 80,000-\$ 99,999$ & 1,319 & $8 \%$ & $\$ 16,497$ & $\$ 2,536$ & $\$ 3,512$ & $\$ 4,846$ \\
\hline$\$ 100,000$ Above & 3,976 & $23 \%$ & $\$ 16,601$ & $\$ 2,520$ & $\$ 3,533$ & $\$ 3,869$ \\
\hline Not Reported & 2,702 & $16 \%$ & $\mathrm{n} / \mathrm{a}$ & $\$ 3,296$ & $\$ 2,730$ & $\$ 153$ \\
\hline TOTAL & 17,086 & $100 \%$ & $\$ 13,918^{*}$ & $\$ 284$ & $\$ 5,695$ & $\$ 3,423$ \\
\hline
\end{tabular}

Notes: This data only represents Fall and Spring financial aid data and is accurate as of March 31, 2016. Please note that small changes to Spring 2016 awards are possible before the data is finalized. Family Income Groups are based on the Total Family Income (including untaxed income) as reported on student FAFSA records. Full-time Students is a headcount based on at least 24 credit hours during Fall and Spring terms. Average Gift Aid includes all grants and scholarships from Federal, State, University and other private sources administered by the Financial Aid Office. Student waivers are also included in the Gift Aid amount. Gift Aid does not include the parental contribution towards EFC. Net Cost of Attendance is the actual average of the total Costs of Attendance (which will vary by income group due to the diversity of students living on- \& off- campus) minus the average Gift Aid amount. Net Tuition \& Fees is the actual average of the total costs of tuition and fees (which will vary by income group due to the amount of credit hours students are enrolled) minus the average Gift Aid amount (see page 16 for list of fees that are included). Average Loan Amount includes Federal (Perkins, Stafford, Ford Direct, and PLUS loans) and all private loans. 'Not Reported' represents the students who did not file a FAFSA. The bottom-line Total/Average represents the average of all full-time undergraduate Florida residents (note*: the total Net Cost of Attendance does not include students who did not report their family income data. 


\section{UNIVERSITY REVENUES}

University Revenues (in Millions of Dollars)

EDUCATION \& GENERAL

\begin{tabular}{|c|c|c|}
\hline Main Operations & & \\
\hline State Funds & $\$ 239.8$ & $\$ 250.3$ \\
\hline Tuition & $\$ 165.7$ & $\$ 169.6$ \\
\hline Subtotal & $\$ 405.5$ & $\$ 419.9$ \\
\hline \multicolumn{3}{|l|}{ Health-Science Center / Medical Schools } \\
\hline State Funds & $\$ 74.7$ & $\$ 74.2$ \\
\hline Tuition & $\$ 50.6$ & $\$ 57.7$ \\
\hline Subtotal & $\$ 125.2$ & $\$ 131.9$ \\
\hline E\&G TOTAL & $\$ 530.8$ & $\$ 551.8$ \\
\hline
\end{tabular}

\section{OTHER BUDGET ENTITIES}

$\begin{array}{lll}\text { Auxiliary Enterprises } & \$ 189.6 & \$ 224.5 \\ \text { Contracts \& Grants } & \$ 278.3 & \$ 384.8 \\ \text { Local Funds } & \$ 429.9 & \$ 477.7 \\ \text { Faculty Practice Plans } & \$ 229.2 & \$ 245.9\end{array}$

Note: State funds include recurring and non-recurring General Revenue funds, Lottery funds appropriated by the Florida Legislature. Actual tuition includes base tuition and tuition differential fee revenues for resident and nonresident undergraduate and graduate students net of waivers. Source: Tables $1 \mathrm{~A} \& 1 \mathrm{E}$ of the annual Accountability Report. 
FINAL - JUNE 2016

\section{UNIVERSITY TUITION, FEES AND HOUSING PROJECTIONS}

\section{University of South Florida - Main Campus}

\begin{tabular}{|c|c|c|c|c|c|c|c|}
\hline \multirow[t]{2}{*}{ Undergraduate Students } & \multicolumn{3}{|c|}{ 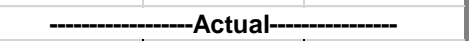 } & \multicolumn{4}{|c|}{ 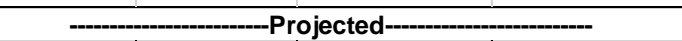 } \\
\hline & 2013-14 & 2014-15 & 2015-16 & 2016-17 & 2017-18 & 2018-19 & 2019-20 \\
\hline \multicolumn{8}{|l|}{ Tuition: } \\
\hline Base Tuition - (0\% inc. for $2016-17$ to $2019-20)$ & $\$ 105.07$ & $\$ 105.07$ & $\$ 105.07$ & $\$ 105.07$ & $\$ 105.07$ & $\$ 105.07$ & $\$ 105.07$ \\
\hline Tuition Differential $^{5}$ & $\$ 46.88$ & $\$ 46.88$ & $\$ 46.88$ & $\$ 46.88$ & $\$ 46.88$ & $\$ 46.88$ & $\$ 46.88$ \\
\hline Total Base Tuition \& Differential per Credit Hour & $\$ 151.95$ & $\$ 151.95$ & $\$ 151.95$ & $\$ 151.95$ & $\$ 151.95$ & $\$ 151.95$ & $\$ 151.95$ \\
\hline$\%$ Change & $1.2 \%$ & $0.0 \%$ & $0.0 \%$ & $0.0 \%$ & $0.0 \%$ & $0.0 \%$ & $0.0 \%$ \\
\hline \multicolumn{8}{|l|}{ Fees (per credit hour): } \\
\hline Student Financial Aid $^{1}$ & $\$ 5.25$ & $\$ 5.25$ & $\$ 5.25$ & $\$ 5.25$ & $\$ 5.25$ & $\$ 5.25$ & $\$ 5.25$ \\
\hline Capital Improvement $^{2}$ & $\$ 6.76$ & $\$ 6.76$ & $\$ 6.76$ & $\$ 6.76$ & $\$ 6.76$ & $\$ 6.76$ & $\$ 6.76$ \\
\hline Activity \& Service & $\$ 12.08$ & $\$ 12.08$ & $\$ 12.08$ & $\$ 12.08$ & $\$ 12.08$ & $\$ 12.08$ & $\$ 12.08$ \\
\hline Health & $\$ 9.94$ & $\$ 10.15$ & $\$ 10.15$ & $\$ 10.15$ & $\$ 10.15$ & $\$ 10.15$ & $\$ 10.15$ \\
\hline Athletic & $\$ 14.46$ & $\$ 14.46$ & $\$ 14.46$ & $\$ 14.46$ & $\$ 14.46$ & $\$ 14.46$ & $\$ 14.46$ \\
\hline Transportation Access & $\$ 3.00$ & $\$ 3.00$ & $\$ 3.00$ & $\$ 3.00$ & $\$ 3.00$ & $\$ 3.00$ & $\$ 3.00$ \\
\hline Technology $^{1}$ & $\$ 5.25$ & $\$ 5.25$ & $\$ 5.25$ & $\$ 5.25$ & $\$ 5.25$ & $\$ 5.25$ & $\$ 5.25$ \\
\hline Marshall Center Fee & $\$ 1.50$ & $\$ 1.50$ & $\$ 1.50$ & $\$ 1.50$ & $\$ 1.50$ & $\$ 1.50$ & $\$ 1.50$ \\
\hline Student Green Energy & $\$ 1.00$ & $\$ 1.00$ & $\$ 1.00$ & $\$ 1.00$ & $\$ 1.00$ & $\$ 1.00$ & $\$ 1.00$ \\
\hline Sub total (credit hour fees) & $\$ 59.24$ & $\$ 59.45$ & $\$ 59.45$ & $\$ 59.45$ & $\$ 59.45$ & $\$ 59.45$ & $\$ 59.45$ \\
\hline Total Tuition and Fees per credit hour & $\$ 211.19$ & $\$ 211.40$ & $\$ 211.40$ & $\$ 211.40$ & $\$ 211.40$ & $\$ 211.40$ & $\$ 211.40$ \\
\hline$\%$ Change & $1.2 \%$ & $0.1 \%$ & $0.0 \%$ & $0.0 \%$ & $0.0 \%$ & $0.0 \%$ & $0.0 \%$ \\
\hline \multicolumn{8}{|l|}{ Fees (block per term): } \\
\hline Activity \& Service & $\$ 7.00$ & $\$ 7.00$ & $\$ 7.00$ & $\$ 7.00$ & $\$ 7.00$ & $\$ 7.00$ & $\$ 7.00$ \\
\hline Health & & - & - & - & - & - & - \\
\hline Athletic & $\$ 10.00$ & $\$ 10.00$ & $\$ 10.00$ & $\$ 10.00$ & $\$ 10.00$ & $\$ 10.00$ & $\$ 10.00$ \\
\hline Transportation Access & & - & & - & - & - & - \\
\hline Marshall Center Fee & $\$ 20.00$ & $\$ 20.00$ & $\$ 20.00$ & $\$ 20.00$ & $\$ 20.00$ & $\$ 20.00$ & $\$ 20.00$ \\
\hline \multicolumn{8}{|l|}{ Propsed NewFee } \\
\hline Total Block Fees per term & $\$ 37.00$ & $\$ 37.00$ & $\$ 37.00$ & $\$ 37.00$ & $\$ 37.00$ & $\$ 37.00$ & $\$ 37.00$ \\
\hline$\%$ Change & $0.0 \%$ & $0.0 \%$ & $0.0 \%$ & $0.0 \%$ & $0.0 \%$ & $0.0 \%$ & $0.0 \%$ \\
\hline Total Tuition for 30 Credit Hours & $\$ 4,558.50$ & $\$ 4,558.50$ & $\$ 4,558.50$ & $\$ 4,558.50$ & $\$ 4,558.50$ & $\$ 4,558.50$ & $\$ 4,558.50$ \\
\hline Total Fees for 30 Credit Hours & $\$ 1,851.20$ & $\$ 1,857.50$ & $\$ 1,857.50$ & $\$ 1,857.50$ & $\$ 1,857.50$ & $\$ 1,857.50$ & $\$ 1,857.50$ \\
\hline Total Tuition and Fees for 30 Credit Hours & $\$ 6,409.70$ & $\$ 6,416.00$ & $\$ 6,416.00$ & $\$ 6,416.00$ & $\$ 6,416.00$ & $\$ 6,416.00$ & $\$ 6,416.00$ \\
\hline \$Change & $\$ 75.30$ & $\$ 6.30$ & $\$ 0.00$ & $\$ 0.00$ & $\$ 0.00$ & $\$ 0.00$ & $\$ 0.00$ \\
\hline$\%$ Change & $1.2 \%$ & $0.1 \%$ & $0.0 \%$ & $0.0 \%$ & $0.0 \%$ & $0.0 \%$ & $0.0 \%$ \\
\hline \multicolumn{8}{|l|}{ Out-of-State Fees } \\
\hline 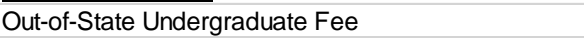 & $\$ 346.50$ & $\$ 346.50$ & $\$ 346.50$ & $\$ 346.50$ & $\$ 346.50$ & $\$ 346.50$ & $\$ 346.50$ \\
\hline Out-of-State Undergraduate Student Financial Aid ${ }^{3}$ & $\$ 17.32$ & $\$ 17.32$ & $\$ 17.32$ & $\$ 17.32$ & $\$ 17.32$ & $\$ 17.32$ & $\$ 17.32$ \\
\hline Total per credit hour & $\$ 363.82$ & $\$ 363.82$ & $\$ 363.82$ & $\$ 363.82$ & $\$ 363.82$ & $\$ 363.82$ & $\$ 363.82$ \\
\hline$\%$ Change & $10 \%$ & $0 \%$ & $0 \%$ & $0 \%$ & $0 \%$ & $0 \%$ & $0 \%$ \\
\hline Total Tuition for 30 Credit Hours & $\$ 4,558.50$ & $\$ 4,558.50$ & $\$ 4,558.50$ & $\$ 4,558.50$ & $\$ 4,558.50$ & $\$ 4,558.50$ & $\$ 4,558.50$ \\
\hline Total Fees for 30 Credit Hours & $\$ 12,765.80$ & $\$ 12,772.10$ & $\$ 12,772.10$ & $\$ 12,772.10$ & $\$ 12,772.10$ & $\$ 12,772.10$ & $\$ 12,772.10$ \\
\hline Total Tuition and Fees for 30 Credit Hours & $\$ 17,324.30$ & $\$ 17,330.60$ & $\$ 17,330.60$ & $\$ 17,330.60$ & $\$ 17,330.60$ & $\$ 17,330.60$ & $\$ 17,330.60$ \\
\hline \$Change & $\$ 1,067.40$ & $\$ 0.00$ & $\$ 0.00$ & $\$ 0.00$ & $\$ 0.00$ & $\$ 0.00$ & $\$ 0.00$ \\
\hline$\%$ Change & $6.6 \%$ & $0.0 \%$ & $0.0 \%$ & $0.0 \%$ & $0.0 \%$ & $0.0 \%$ & $0.0 \%$ \\
\hline Housing/Dining $^{4}$ & $\$ 9,250.00$ & $\$ 9,403.00$ & $\$ 9,403.00$ & $\$ 10,324.00$ & $\$ 11,074.00$ & $\$ 11,800.00$ & $\$ 11,800.00$ \\
\hline \$ Change & & $\$ 153.00$ & $\$ 0.00$ & $\$ 921.00$ & $\$ 750.00$ & $\$ 726.00$ & $\$ 0.00$ \\
\hline$\%$ Change & & $1.7 \%$ & $0.0 \%$ & $9.8 \%$ & $7.3 \%$ & $6.6 \%$ & $0.0 \%$ \\
\hline \multicolumn{8}{|l|}{${ }^{2}$ as approved by the Board of Governors. } \\
\hline \multicolumn{8}{|l|}{${ }^{3}$ can be no more than $5 \%$ of tuition and the out-of-state fee. } \\
\hline \multicolumn{8}{|c|}{${ }^{4}$ combine the most popular housing and dining plans provided to students } \\
\hline${ }^{5}$ report current tuition differential. Only UF or FSU can re & ct potential incr & es & & & & & \\
\hline
\end{tabular}

Note: The University will continually assess the need for changes to tuition \& fees in support of student success and fiscal responsibility consistent with FL Statute 1009.24 


\section{DEFINITIONS}

\section{Performance Based Funding}

Percent of Bachelor's Graduates Enrolled or Employed $(\$ 25,000+)$ in the U.S. One Year After Graduation

\section{Median Wages \\ of Bachelor's Graduates Employed Full-time in Florida One Year After Graduation}

This metric is based on the percentage of a graduating class of bachelor's degree recipients who are enrolled or employed (earning at least $\$ 25,000$ ) somewhere in the United States. Students who do not have valid social security numbers and are not found enrolled are excluded. Note: This data now non-Florida employment data.

Sources: State University Database System (SUDS), Florida Education \& Training Placement Information Program (FETPIP) analysis of Wage Record Interchange System (WRIS2) and Federal Employment Data Exchange (FEDES), and National Student Clearinghouse (NSC).

This metric is based on annualized Unemployment Insurance (UI) wage data from the fourth fiscal quarter after graduation for bachelor's recipients. UI wage data does not include individuals who are self-employed, employed out of state, employed by the military or federal government, those without a valid social security number, or making less than minimum wage. Sources: State University Database System (SUDS), Florida Education \& Training Placement Information Program (FETPIP), National Student Clearinghouse.

For each of the last four years of data, the annual undergraduate total full expenditures (includes direct and indirect expenditures) were divided by the total fundable student credit hours to create a cost per credit hour for each year. This cost per credit hour was then multiplied by 30 credit hours to derive an average annual cost. The average annual cost for each of the four years was summed to provide an average cost per degree for a baccalaureate degree that requires 120 credit hours. Sources: State University Database System (SUDS), Expenditure Analysis: Report IV.

Six Year FTIC Graduation Rate
This metric is based on the percentage of first-time-in-college (FTIC) students who started in the Fall (or summer continuing to Fall) term and had graduated from the same institution within six years. Source: Accountability Report (Table 4D).

\section{Academic \\ Progress Rate \\ 2nd Year Retention \\ with GPA Above 2.0}

This metric is based on the percentage of first-time-in-college (FTIC) students who started in the Fall (or summer continuing to Fall) term and were enrolled full-time in their first semester and were still enrolled in the same institution during the Fall term following their first year with had a grade point average (GPA) of at least 2.0 at the end of their first year (Fall, Spring, Summer). Source: Accountability Report (Table 4B).
University Access Rate Percent of Undergraduates with a Pell-grant

\section{Bachelor's Degrees within Programs of Strategic Emphasis}

\section{Graduate Degrees within Programs of Strategic Emphasis}

This metric is based the number of undergraduates, enrolled during the fall term, who received a Pell-grant during the fall term. Unclassified students, who are not eligible for Pellgrants, were excluded from this metric. Source: Accountability Report (Table 3E).

This metric is based on the number of baccalaureate degrees awarded within the programs designated by the Board of Governors as 'Programs of Strategic Emphasis'. A student who has multiple majors in the subset of targeted Classification of Instruction Program codes will be counted twice (i.e., double-majors are included). Source: Accountability Report (Table $4 \mathrm{H})$.

This metric is based on the number of graduate degrees awarded within the programs designated by the Board of Governors as 'Programs of Strategic Emphasis'. A student who has multiple majors in the subset of targeted Classification of Instruction Program codes will be counted twice (i.e., double-majors are included). Source: Accountability Report (Table $5 C)$. 


\section{BOG Choice Metrics}

\section{Percent of Bachelor's Degrees Without Excess Hours}

This metric is based on the percentage of baccalaureate degrees awarded within $110 \%$ of the credit hours required for a degree based on the Board of Governors Academic Program Inventory.

Note: It is important to note that the statutory provisions of the "Excess Hour Surcharge" (1009.286, FS) have been modified several times by the Florida Legislature, resulting in a phased-in approach that has created three different cohorts of students with different requirements. The performance funding metric data is based on the latest statutory requirements that mandates $110 \%$ of required hours as the threshold. In accordance with statute, this metric excludes the following types of student credits (ie, accelerated mechanisms, remedial coursework, non-native credit hours that are not used toward the degree, non-native credit hours from failed, incomplete, withdrawn, or repeated courses, credit hours from internship programs, credit hours up to 10 foreign language credit hours, and credit hours earned in military science courses that are part of the Reserve Officers' Training Corps (ROTC) program). Source: State University Database System (SUDS).

\section{BOT Choice Metrics}

Number of Postdoctoral
Appointees
USF

This metric is based on the number of post-doctoral appointees at the beginning of the academic year. A postdoctoral researcher has recently earned a doctoral (or foreign equivalent) degree and has a temporary paid appointment to focus on specialized research/scholarship under the supervision of a senior scholar.

Source: National Science Foundation/National Institutes of Health annual Survey of Graduate Students and Postdoctorates in Science and Engineering (GSS).

\section{Preeminent Research University Funding Metrics}

\section{Average GPA and SAT Score}

\section{Ranking}

An average weighted grade point average of 4.0 or higher and an average SAT score of 1200 or higher for fall semester incoming freshmen, as reported annually in the admissions data that universities submit to the Board of Governors. This data includes registered FTIC (student type='B','E') with an admission action of admitted or provisionally admitted ('A','P', 'X').

A top-50 ranking on at least two well-known and highly respected national public university rankings, reflecting national preeminence, using most recent rankings, includes: Princeton Review, Fiske Guide, QS World University Ranking, Times Higher Education World University Ranking, Academic Ranking of World University, US News and World Report National University, US News and World Report National Public University, US News and World Report Liberal Arts Colleges, Forbes, Kiplinger, Washington Monthly Liberal Arts Colleges, Washington Monthly National University, and Center for Measuring University Performance. 
Freshman Retention Rate (Full-time, FTIC)
Freshman Retention Rate (Full-time, FTIC) as reported annually to the Integrated Postsecondary Education Data System (IPEDS). The retention rates that are reported in the Board's annual Accountability report are preliminary because they are based on student enrollment in their second fall term as reported by the 28th calendar day following the first day of class. When the Board of Governors reports final retention rates to IPEDS in the Spring (usually the first week of April), that data is based on the student enrollment data as reported after the Fall semester has been completed. The preliminary and final retention rates are nearly identical when rounded to the nearest whole number.

Cohorts are based on undergraduate students who enter the institution in the Fall term (or Summer term and continue into the Fall term). Percent Graduated is based on federal rate and does not include students who originally enroll as part-time students, or who transfer

6-year Graduation Rate (Full-time, FTIC) into the institution. This metric complies with the requirements of the federal Student Right to Know Act that requires institutions to report the completion status at $150 \%$ of normal time (or six years). For more information about how this data is calculated, see: http://www.flbog.edu/about/budget/docs/performance_funding/PBF_GRADUATION_and_RETENTIO N_Methodology_FINAL.pdf.

National Academy Memberships held by faculty as reported by the Center for Measuring University Performance in the Top American Research Universities (TARU) annual report or the official membership directories maintained by each national academy.

Science \& Engineering Research Expenditures, including federal research expenditures as reported annually to the National Science Foundation (NSF).

\section{Science \& Engineering Research Expenditures (\$M)}

Non-Medical Science \& Engineering Research Expenditures (\$M)

National Ranking in S.T.E.M. Research Expenditures

Patents Awarded

(3 calendar years)
Total S\&E research expenditures in non-medical sciences as reported to the NSF. This removes medical sciences funds (9F \& $12 F$ in HERD survey) from the total S\&E amount.

The NSF identifies 8 broad disciplines within Science \& Engineering (Computer Science, Engineering, Environmental Science, Life Science, Mathematical Sciences, Physical Sciences, Psychology, Social Sciences). The rankings by discipline are determined by BOG staff using the NSF WebCaspar database.

Total patents awarded by the United States Patent and Trademark Office (USPTO) for the most recent three calendar year period. Due to a year-lag in published reports, Board of Governors staff query the USPTO database with a query that only counts utility patents:"(AN/"University Name" AND ISD/yyyymmdd->yyyymmdd AND APT/1)".

Doctoral Degrees Awarded Annually

\section{Number of Post-Doctoral Appointees}

Endowment Size (\$M)
Doctoral degrees awarded annually, as reported annually in the Board of Governors Accountability Report.

The number of Postdoctoral Appointees awarded annually, as reported in the TARU annual report. This data is based on National Science Foundation/National Institutes of Health annual Survey of Graduate Students and Postdoctorates in Science and Engineering (GSS).

This data comes from the National Association of College and University Business Officers (NACUBO) and Commonfund Institute's annual report of Market Value of Endowment Assets - which, due to timing, may release the next fiscal year's data after the Board of Governors Accountability report is published. 


\section{Key Performance Indicators \\ Teaching \& Learning Metrics}

Freshmen in Top 10\%

of HS Graduating Class

Professional/Licensure

Exam First-time Pass Rates

Average Time to Degree

Mean Years for FTIC

in $120 \mathrm{hr}$ programs

FTIC Graduation Rates

In 4 years (or less)

Bachelor's Degrees Awarded

Graduate Degrees Awarded

Bachelor's Degrees Awarded To African-American and Hispanic Students

Percent of all degree-seeking, first-time, first-year (freshman) students who had high school class rank within the top $10 \%$ of their graduating high school class. As reported by the university to the Common Data Set (C10).

The number of exams with first-time pass rates above and below the national or state average, as reported in the annual Accountability report, including: Nursing, Law, Medicine (3 subtests), Veterinary, Pharmacy, Dental (2 subtests), Physical Therapy, and Occupational Therapy.

This metric is the mean number of years between the start date (using date of most recent admission) and the end date (using the last month in the term degree was granted) for a graduating class of first-time, single-major baccalaureates in 120 credit hour programs within a (Summer, Fall, Spring) year.

As reported in the annual Accountability report (table 4D), First-time-in-college (FTIC) cohort is defined as undergraduates entering in fall term (or summer continuing to fall) with fewer than 12 hours earned since high school graduation. The rate is the percentage of the initial cohort that has either graduated from or is still enrolled in the same institution by the fourth academic year. Both full-time and part-time students are used in the calculation. The initial cohort is revised to remove students, who have allowable exclusions as defined by IPEDS, from the cohort.

This is a count of baccalaureate degrees awarded as reported in the annual Accountability Report (Table 4G).

This is a count of graduate degrees awarded as reported in the Accountability Report (Table $5 B)$.

Non-Hispanic Black and Hispanic do not include students classified as Non-Resident Alien or students with a missing race code - as reported in the Accountability Report (table 4I).

Students who earn two distinct degrees in the same term are counted twice - whether their degrees are from the same six-digit CIP code or different CIP codes. Students who earn only one degree are counted once - even if they completed multiple majors or tracks. Percentage of Degrees is based on the number of baccalaureate degrees awarded to non-Hispanic Black and Hispanic students divided by the total degrees awarded - excluding those awarded to non-resident aliens and unreported.

This metric is based on the age of the student at the time of enrollment (not upon entry). Age acts as a surrogate variable that captures a large, heterogeneous population of adult students who often have family and work responsibilities as well as other life circumstances that can interfere with successful completion of educational objectives.

Full-time Equivalent (FTE) student is a measure of instructional activity that is based on the number of credit hours that students enroll. FTE is based on the US definition, which divides undergraduate credit hours by 30 . Distance Learning is a course in which at least 80 percent of the direct instruction of the course is delivered using some form of technology when the student and instructor are separated by time or space, or both (per 1009.24(17), F.S.).

The percentage of baccalaureate degrees that are classified as STEM by the Board of Governors in the SUS program inventory as reported in the annual Accountability Report (Table 4H).

Percent of Bachelor's Degrees in STEM \& Health

Percent of Graduate Degrees in STEM \& Health

The percentage of baccalaureate degrees that are classified as STEM by the Board of Governors in the SUS program inventory as reported in the annual Accountability Report (Table 5C). 


\section{Key Performance Indicators (continued) \\ Scholarship, Research \& Innovation Metrics}

Awards include: American Council of Learned Societies (ACLS) Fellows, Beckman Young Investigators, Burroughs Wellcome Fund Career Awards, Cottrell Scholars, Fulbright American Scholars, Getty Scholars in Residence, Guggenheim Fellows, Howard Hughes Medical Institute Investigators, Lasker Medical Research Awards, MacArthur Foundation Fellows, Andrew W. Mellon Foundation Distinguished Achievement Awards, National

Faculty Awards Endowment for the Humanities (NEH) Fellows, National Humanities Center Fellows, National Institutes of Health (NIH) MERIT, National Medal of Science and National Medal of Technology, NSF CAREER awards (excluding those who are also PECASE winners), Newberry Library Long-term Fellows, Pew Scholars in Biomedicine, Presidential Early Career Awards for Scientists and Engineers (PECASE), Robert Wood Johnson Policy Fellows, Searle Scholars, Sloan Research Fellows, Woodrow Wilson Fellows. As reported by the Top American Research Universities - see: http://mup.asu.edu/research data.html.

Total Research Expenditures (\$M)

Percent of R\&D Expenditures funded from External Sources

Licenses/Options Executed
Total expenditures for all research activities (including non-science and engineering activities) as reported in the National Science Foundation annual survey of Higher Education Research and Development (HERD).

This metric reports the amount of research expenditures that was funded from federal, private industry and other (non-state and non-institutional) sources.

Source: National Science Foundation annual survey of Higher Education Research and Development (HERD).

Licenses/options executed in the fiscal year for all technologies as reported in the annual Accountability Report (table 6A).

Number of Start-up Companies

The number of start-up companies that were dependent upon the licensing of University technology for initiation as reported in the annual Accountability Report (table 6A). 


\section{Student Debt Summary}

\section{Percent of Bachelor's Recipients with Debt}

\section{Average Amount of Debt for Bachelor's who have graduated with debt}

This is the percentage of bachelor's graduates in a given academic year who entered the university as a first-time-in-college (FTIC) student and who borrowed through any loan programs (institutional, state, Federal Perkins, Federal Stafford Subsidized and unsubsidized, private) that were certified by your institution - excludes parent loans. Source: Common Dataset $(\mathrm{H} 4)$.

This is the average amount of cumulative principal borrowed (from any loan program certified by the institution) for each native, FTIC bachelor's recipient in a given academic year that graduated with debt - see metric definition above. This average does NOT include students who did not enter a loan program that was certified by the institution. Source: Common Dataset (H5).

Student loan cohort default rate (CDR) data includes undergraduate and graduate students, and refers to the three federal fiscal year period when the borrower enters repayment and ends on the second fiscal year following the fiscal year in which the borrower entered repayment. Cohort default rates are based on the number of borrowers who enter repayment, not the number and type of loans that enter repayment. A borrower with multiple loans from the same school whose loans enter repayment during the same cohort fiscal year will be included in the formula only once for that cohort fiscal year. Default rate debt includes: Federal Stafford Loans, and Direct Stafford/Ford Loans - for more information see: http://ifap.ed.gov/DefaultManagement/CDRGuideMaster.html.

\begin{tabular}{|c|c|c|c|}
\hline $\begin{array}{l}\text { Cohort } \\
\text { Fiscal } \\
\text { Year }\end{array}$ & $\begin{array}{c}\text { Year } \\
\text { Published }\end{array}$ & $\frac{\text { Borrowers in the Numerator }}{\text { Borrowers in the Denominator }}$ & $\begin{array}{l}\frac{\text { 3-Yr Time Period }}{\text { (Numerator) }} \\
\text { 1-Yr Time Period } \\
\text { (Denominator) }\end{array}$ \\
\hline 2009 & 2012 & $\begin{array}{l}\text { Borrowers who entered repayment in } 2009 \\
\text { and defaulted in } 2009,2010 \text { or } 2011 \\
\text { Borrowers who entered repayment in } 2009\end{array}$ & $\frac{10 / 01 / 2008 \text { to } 9 / 30 / 2011}{10 / 01 / 2008 \text { to } 9 / 30 / 2009}$ \\
\hline 2010 & 2013 & $\begin{array}{l}\text { Borrowers who entered repayment in } 2010 \\
\text { and defaulted in } 2010,2011 \text { or } 2012 \\
\text { Borrowers who entered repayment in } 2010\end{array}$ & $\frac{10 / 01 / 2009 \text { to } 9 / 30 / 2012}{10 / 01 / 2009 \text { to } 9 / 30 / 2010}$ \\
\hline 2011 & $2014^{*}$ & $\begin{array}{l}\text { Borrowers who entered repayment in } 2011 \\
\text { and defaulted in } 2011,2012 \text { or } 2013 \\
\text { Borrowers who entered repayment in } 2011\end{array}$ & $\frac{10 / 01 / 2010 \text { to } 9 / 30 / 2013}{10 / 01 / 2010 \text { to } 9 / 30 / 2011}$ \\
\hline 2012 & 2015 & $\begin{array}{l}\text { Borrowers who entered repayment in } 2012 \\
\text { and defaulted in 2012, } 2013 \text { or } 2014 \\
\text { Borrowers who entered repayment in } 2012\end{array}$ & $\frac{10 / 01 / 2011 \text { to } 9 / 30 / 2014}{10 / 01 / 2011 \text { to } 9 / 30 / 2012}$ \\
\hline 2013 & 2016 & $\begin{array}{l}\text { Borrowers who entered repayment in } 2013 \\
\text { and defaulted in } 2013,2014 \text { or } 2015 \\
\text { Borrowers who entered repayment in } 2013\end{array}$ & $\frac{10 / 01 / 2012 \text { to } 9 / 30 / 2015}{10 / 01 / 2012 \text { to } 9 / 30 / 2013}$ \\
\hline 2014 & 2017 & $\begin{array}{l}\text { Borrowers who entered repayment in } 2014 \\
\text { and defaulted in } 2014,2015 \text { or } 2016 \\
\text { Borrowers who entered repayment in } 2014\end{array}$ & $\frac{10 / 01 / 2013 \text { to } 9 / 30 / 2016}{10 / 01 / 2013 \text { to } 9 / 30 / 2014}$ \\
\hline 2015 & 2018 & $\begin{array}{l}\text { Borrowers who entered repayment in } 2015 \\
\text { and defaulted in } 2015,2016 \text { or } 2017 \\
\text { Borrowers who entered repayment in } 2015\end{array}$ & $\frac{10 / 01 / 2014 \text { to } 9 / 30 / 2017}{10 / 01 / 2014 \text { to } 9 / 30 / 2015}$ \\
\hline
\end{tabular}

